\title{
Defective Edge Detection System Design for Long Bamboo Batten
}

\author{
Tian SUN, Xiangwei MOU*, Shuxiang SONG, Jianhui LI \\ College of Electronic Engineering \\ Guangxi Normal University \\ Guilin, China \\ *E-mail: xwmou@gxnu.edu.cn \\ +* Corresponding author
}

\begin{abstract}
To effectively and accurately detect edge defects in bamboos, a defective edge detection system for a long bamboo batten was designed in this study. There is a significant gap difference between the defective and intact edges of bamboo battens. Specifically, a large gap exists between the edge of a defective bamboo batten and a contact plate, whereas the gap is either very small or non-existent for a non-defective bamboo batten. The intensity of the light passing through the gap is detected using an optical fiber amplifier, and an infrared photoelectric switch positions the bamboo. The system can detect the four edges of multiple bamboo battens in real-time under the supervision of a master controller. The reliability of the system was verified through a series of physical experiments. This system can help in improving the production efficiency of bamboo plate processing enterprises, which is of considerable significance for the automatic production of bamboo products.
\end{abstract} batten

Keywords-defective edge detection; fiber amplifier; bamboo

\section{INTRODUCTION}

Sorting bamboo battens is crucial in the production of bamboo products. Workers have to screen bamboo battens with different colors and defects during the sorting process. Screening bamboo battens with defective edges is very timeconsuming, as workers have to carefully observe both sides for detecting defects. This sorting process is much slower than screening by color or by observing the damage caused by worms, which have more obvious characteristics [1-2]; it has become a very unfavorable process that restricts the production efficiency of bamboo sheet processing enterprises.

In recent years, both locally and internationally, research on bamboo defect detection methods has been mainly based on machine vision, such as otsu, sobel, wavelet removal, texture of bamboo batten, and other methods that change bamboo images to binarized images and then find their eigenvalues to detect damage caused by worms, mildew, cracks, bevels, oblique cuts, and triangular strips in the bamboo [3-7]. Researchers have also proposed the use of lasers to determine the damage and defects caused by worms in bamboo chopsticks [8].

However, the above method for the detection of defective bamboo edges still has the following problems: it is effective when the characteristic value of the detected object changes significantly, but is not applicable to the detection of small changes in the characteristic value of the edges of bamboo battens. Fig. 1 shows the defective edges of the bamboo batten. Detecting defects in bamboo chopsticks with laser is also not suitable for bamboos. Considering these problems, we designed a defective edge detection system for a bamboo batten in this study; the system consists of a mechanical structure, master controller, and software testing programs.

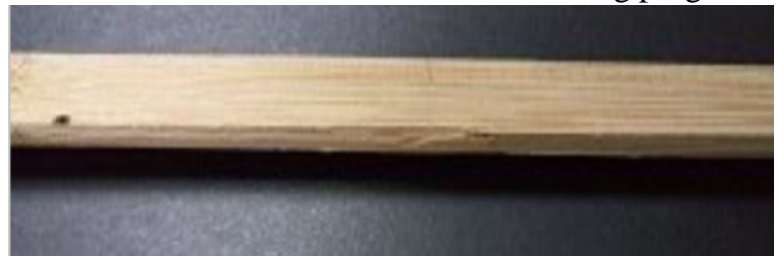

Figure 1. Defective edges of bamboo batten

\section{Machine StRUcture AND Operating PrinciPle}

\section{A. Machine Structure}

As shown in Fig. 2, the defective edge detection system consists of input and output gears, five sets of positioning gears that hold down and position the bamboo batten, and four sets of limit gears, motor, and frame. The input gear, multiple sets of positioning gears, and output gear are arranged in a straight line at the top of the frame. An infrared photoelectric switch is installed on the side of the entrance of the input gear. The four sets of limit gear are installed adjacent to the two sets of positioning gear; the limit gears make contact with the edge of the bamboo batten, and each set of limit gear is equipped with a fiber amplifier near the end of the bamboo batten. The motor is placed at the bottom on the inside of the frame and is connected to the input gear via a drive chain to drive the input gear to input the bamboo battens.

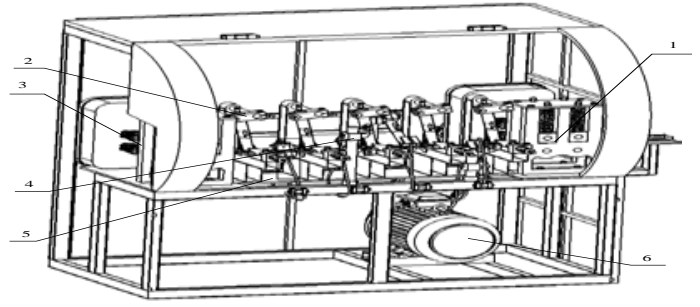

Figure 2. Structure of the defective edge detection system for the bamboo batten: 1: Input gear; 2: Positioning gear; 3: Output gear; 4: Limit gear; 5: Frame; 6: Motor. 
The positioning gear includes a bracket, bottom plate, horizontal elastic lever, and vertical elastic lever. The upper end of the bracket is installed on the bottom plate to convey the bamboo batten, one end of which is an arc transition surface close to the input gear. A supporting lever is fixed on the edge of the upper end of the bracket, and a photoelectric switch is installed on the supporting lever. A vertical elastic lever is arranged above the bottom plate, and the upper part of the vertical elastic lever is connected to the upper part of the supporting lever through a connecting lever. The lower end of the vertical elastic lever is installed with an upper roller that holds the bamboo batten in the vertical direction on the bottom plate. A right roller is installed on one side of the bottom plate, and a horizontal elastic lever is installed on the other side of the bottom plate. The left roller rolls and presses in the horizontal direction of the bamboo batten near one end of the bottom plate.

The limit gear includes an installation seat, a limit elastic lever, and a support frame. The installation seat is affixed to the top of the frame, the lower end of the limit elastic lever is connected to the installation seat by the gripping device, and the upper end is hinged with the support frame; the hinge diagram is shown in Fig. 3. One end of the support frame, away from the limit elastic lever, has a contact plate that makes contact with the two edges of the bamboo batten and is at a 45-degree angle with the bamboo batten. The transmitting end of the fiber amplifier is arranged on the upper end of the support frame through an installation plate, and the receiving end is arranged on the lower end of the support frame through the installation plate. The transmitting end of the fiber amplifier emits a beam to the receiving end of the fiber amplifier.

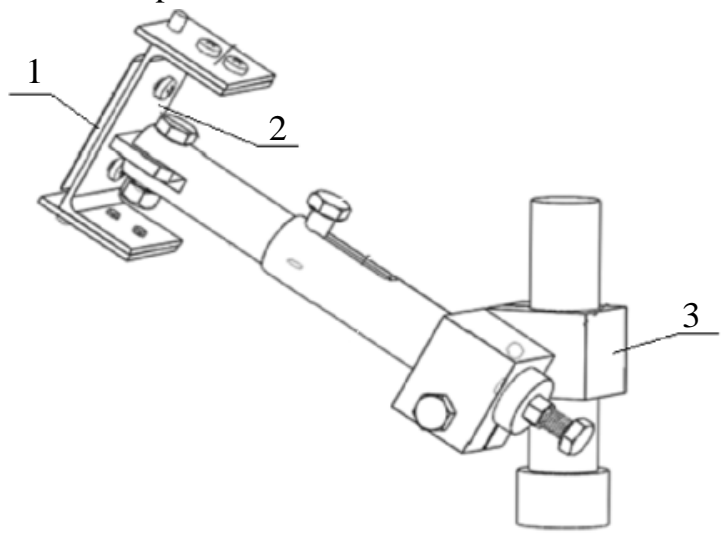

Figure 3. Diagram of the hinge: 1: contact plate; 2: support frame; 3: installation seat.

The structure of the horizontal elastic lever, vertical elastic lever, and the limit elastic lever is identical; they all include a pipe, bolts, a lever, and spring. The spring is compressed in the pipe, and the lever extends into the pipe and makes contact with the spring. A chute is installed at the location where the lever is connected to the pipe; a bolt is arranged in the chute, and the bolt and lever extend into the pipe and are fixed inside the pipe. The lever drives the bolt to slide in the chute. The details of the defective edge detection system for the bamboo batten are depicted in Fig. 4.

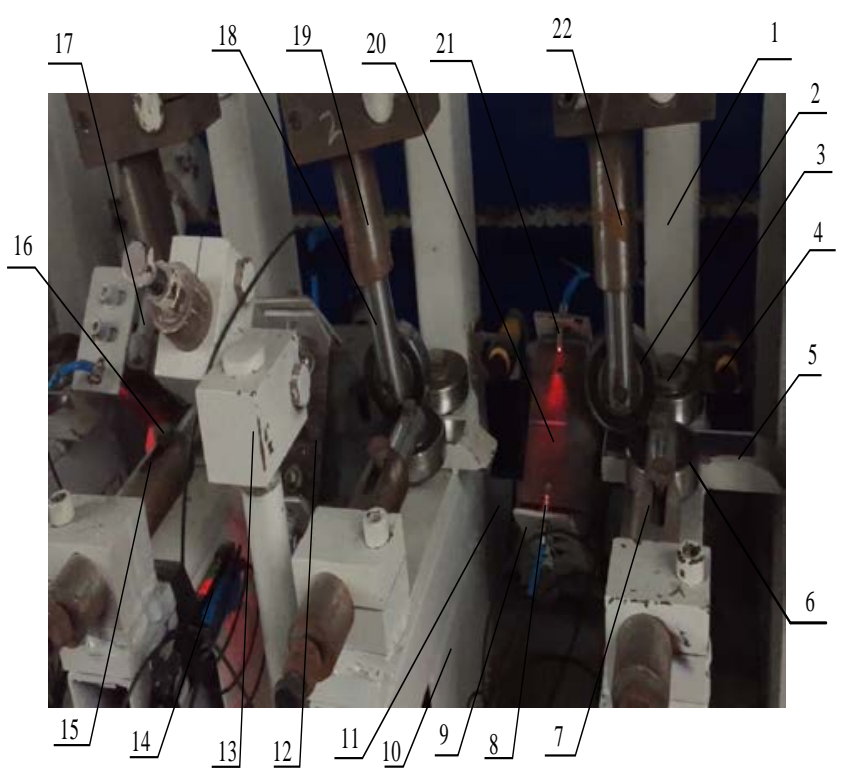

Figure 4. Details of the defective edge detection system for bamboo

batten: 1: Support lever; 2: Upper roller; 3: Right roller; 4: Infrared photoelectric switch; 5: Bottom plate; 6: Left roller; 7: Horizontal elastic lever; 8: Receiving end of fiber amplifier; 9: Installation plate; 10: Bracket; 11: Frame; 12: Support frame; 13: Installation seat; 14: Fiber amplifier; 15: Chute; 16: Bolt; 17: Limit elastic lever; 18: Lever; 19: Pipe; 20: Contact plate; 21: Transmitting end of fiber amplifier; 22: Vertical elastic lever.

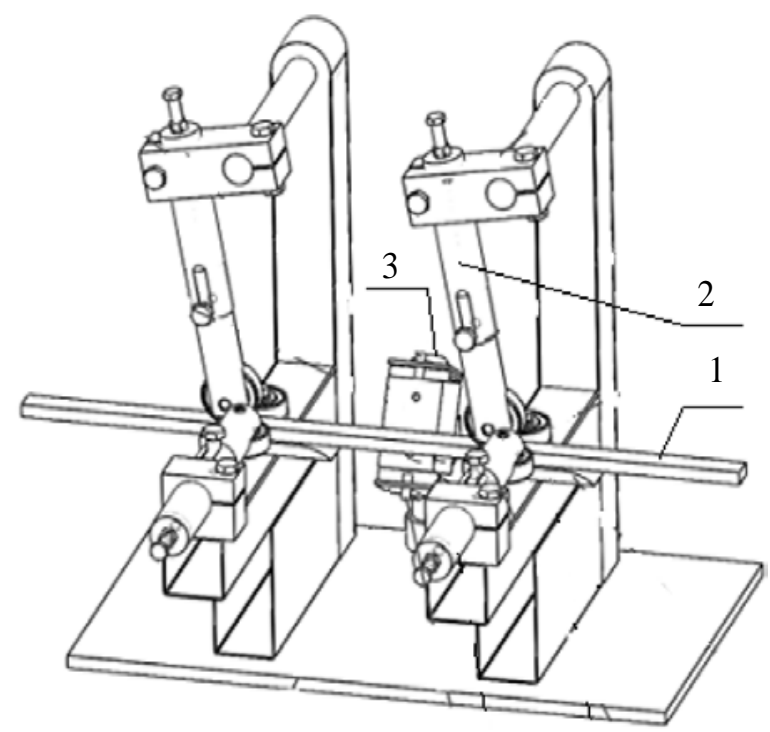

Figure 5. Positioning gear, limit gear, and bamboo batten. 1: Bamboo batten; 2: Positioning gear; 3: Limit gear.

\section{B. Principle of Operation}

According to the transported direction of the bamboo batten, the four sets of limit gear are installed on the lowerright, lower-left, upper-right, and upper-left of the detecting gear in the defective edge detection system. The bamboo batten is pressed and limited by each limit gear, such that the edges of the bamboo batten are tightly connected to the contact plate. In the positioning gear, the vertical elastic lever drives the upper roller in the vertical direction and holds 
down the bamboo batten. The horizontal elastic lever drives the left roller in the horizontal direction and holds down the bamboo batten. The vertical horizontal elastic levers can elastically adjust the upper roller and left roller, respectively. Furthermore, the degree of adjustment is dependent on the contact and the change in status of the shape and size of the bamboo batten. The positioning gear and limit gears hold down and copy the edge of the bamboo batten, which is shown in Fig. 5. Through cooperation between these two gears, all types of deformed bamboo battens can be stably transported through the system, and these two gears work simultaneously to make a defective edge produce a large gap and an intact edge produce a small gap when contacting the contact plate, as shown in Fig. 6.

(a)

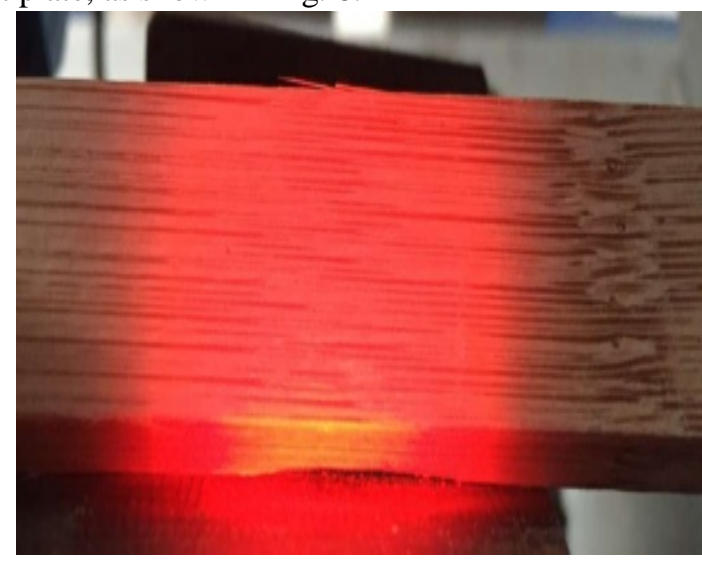

(b)

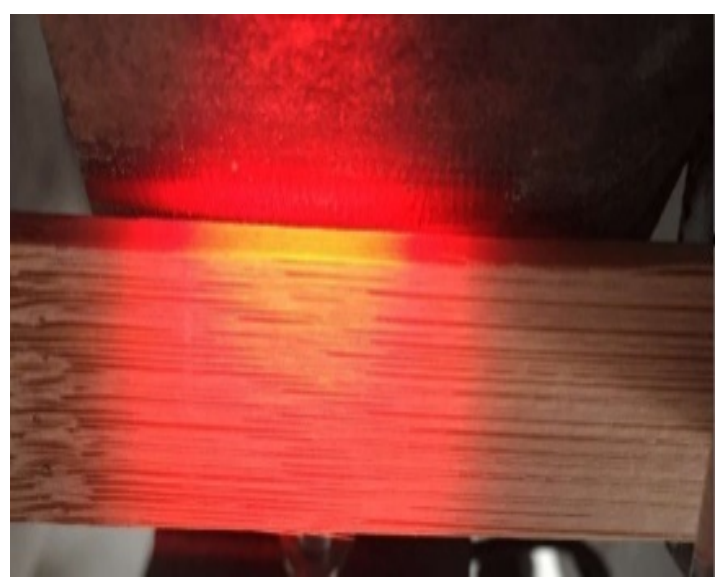

Figure 6. Gap between the bamboo and contact plate: (a) The bamboo batten with a defective edge produces a large gap on the contact plate. (b): The bamboo batten with an intact edge produces a smaller gap on the contact plate $\mathrm{G}$

\section{Detection Process}

When the bamboo batten enters the defect detection system, the infrared photoelectric switch is used to locate the bamboo batten, and when the bamboo batten reaches the contact plate, the master controller starts to detect the signal of the fiber amplifier. When the fiber amplifier detects a bamboo batten with defective edges, a feedback signal is sent to the master controller. Conversely, when the bamboo batten passes through the detection gear, the fiber amplifier does not receive a feedback signal, and the main controller marks the bamboo batten as having its edge intact.

\section{Master Controller Design}

The master controller uses STM32 as the main control chip. This chip is connected to the infrared photoelectric switch and optical fiber amplifier and is connect to the host computer through a serial port. The master controller consists of a power supply circuit, serial port circuit, photoelectric switch signal receiving circuit, optical fiber amplifier signal, and receiving circuit. Fig. 5 shows the master controller.

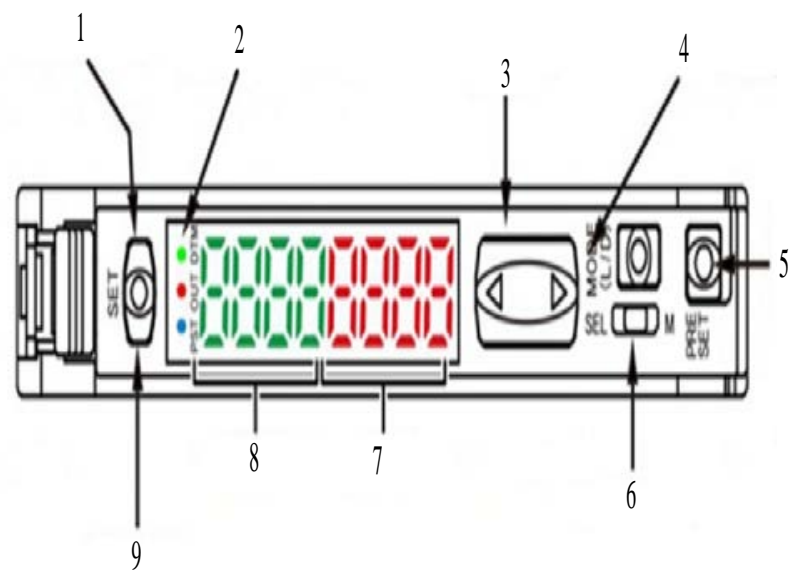

Figure 7. ER2-31H double digital display fiber amplifier. 1: Set button; 2 DTM, PST (working status indicator lights.) OUT: Feedback signal indicator light; 3: Sensitivity fine tune; 4: Mode/Output; 5: Preset function; 6: MEGA Selector switch; 7: Light intensity value; 8: Setting value.

\section{E. Selection of Fiber Amplifier}

The BOJKE Boyi Mini ER2-31H double digital display fiber amplifier is the device used to detect defects at the edge of the bamboo batten. The fastest response speed of ER2$31 \mathrm{H}$ is 40 us; it has a three-wire mode (two power lines and a signal line to ground) and allows manual setting of thresholds. When the fiber amplifier detects that the light intensity value exceeds the threshold, a feedback voltage signal is generated. Fig. 7 shows the ER2-31H double digital display fiber amplifier components and their functions.

\section{F. Fiber Amplifier Threshold Settings}

The threshold setting of the fiber amplifier has a direct relationship with the accuracy with which the defective edges of the bamboo batten are detected. The setting of the threshold is also related to the mechanical mechanism and environmental factors. Through the commissioning of the entire machine, 25 representative experiments were carried out. The threshold of fiber amplifier was tested by marking the defect and intact edges of the bamboo with a "1" and "2," respectively. As shown in Table I, the fiber amplifier detected the defective edges of the bamboo battens, for which the values are higher than 600. For the detection of intact bamboo batten edges, the values are less than 600 . Therefore, set the threshold to 600 . 
TABLE I. FiBer AMPLIFIER DETECTION VALUeS

\begin{tabular}{|c|c|c|}
\hline Number & $\begin{array}{c}\text { Category of bamboo } \\
\text { batten }\end{array}$ & $\begin{array}{c}\text { Fiber amplifier } \\
\text { value }\end{array}$ \\
\hline 1 & 1 & $75-223$ \\
\hline 2 & 1 & 56-85 \\
\hline 3 & 1 & $53-435$ \\
\hline 4 & 1 & $113-546$ \\
\hline 5 & 1 & $32-110$ \\
\hline 6 & 1 & $57-239$ \\
\hline 7 & 1 & $16-79$ \\
\hline 8 & 1 & $25-356$ \\
\hline 9 & 1 & $36-469$ \\
\hline 10 & 1 & $1674-3541$ \\
\hline 11 & 2 & $2145-3228$ \\
\hline 12 & 2 & $1180-1974$ \\
\hline 13 & 2 & $1341-3742$ \\
\hline 14 & 2 & 863-2649 \\
\hline 15 & 2 & $1452-3230$ \\
\hline 16 & 2 & $1323-5214$ \\
\hline 17 & 2 & $752-2428$ \\
\hline 18 & 2 & $1570-3576$ \\
\hline 19 & 2 & $1846-5781$ \\
\hline 20 & 2 & $723-1750$ \\
\hline 21 & 2 & $1816-3577$ \\
\hline 22 & 2 & $1445-3956$ \\
\hline 23 & 2 & $947-2565$ \\
\hline 24 & 2 & $3597-5247$ \\
\hline 25 & 2 & $2360-5610$ \\
\hline
\end{tabular}

\section{SOFTWARE DESIGN}

The length of the bamboo batten used industrially is approximately $2.2 \mathrm{~m}$, whereas the length of the defective edge of the bamboo batten detection machine is approximately $3.2 \mathrm{~m}$. Therefore, it is only necessary to consider two bamboo battens in the system at the same time. To this end, the test program set the parity flag, used to represent odd bamboo and even bamboo, respectively. Each group of odd (even) detection programs had a corresponding defect-parameter D1 (D2).

The defective edge detection program flowchart for four program groups is shown in Fig. 8. In each group of defective edge detection programs, when a falling edge signal is indicated by the corresponding fiber amplifier, a determination is made as to whether the corresponding photoelectric switch $=1$. If both conditions are met, the odd (even) defect-parameter D1 (D2) is incremented by one. Conversely, if the above two judgments have one condition that is not met, the program will return. In the upper-right corner detection program, when a falling edge signal is indicated by the lower-right photoelectric switch, the program executes a reverse parity flag. Then, when the falling edge of the signal is indicated by the upper-left photoelectric switch, the program will determine whether the defect-parameter D1 $(\mathrm{D} 2)=0$. If parameter D1 $(\mathrm{D} 2)=0$, the program marks the current bamboo batten as an intact edge bamboo batten; otherwise, it marks it as a defective edge bamboo batten.

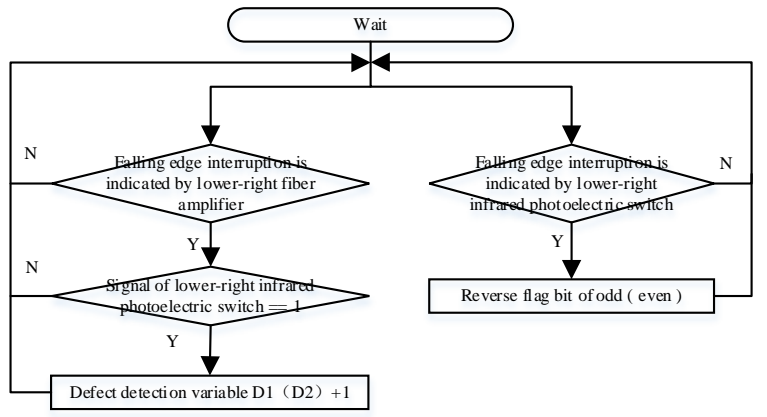

(a)

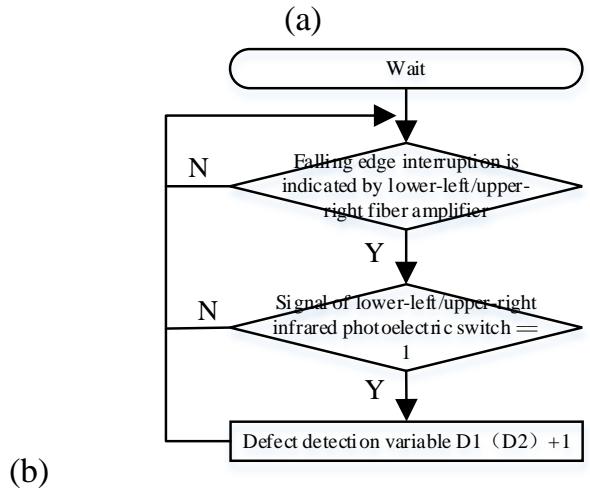

(b)

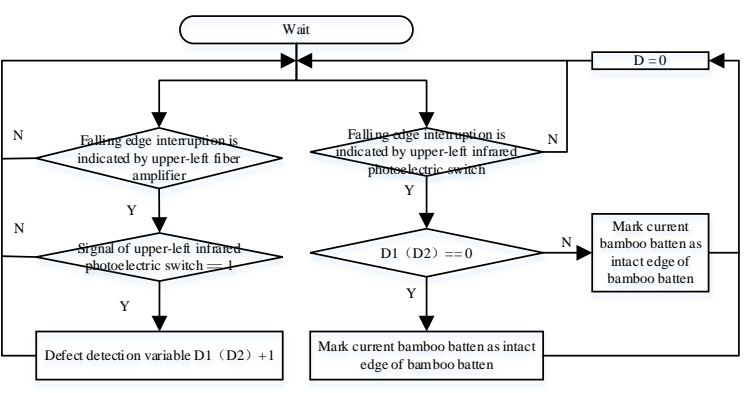

(c)

Figure 8. Flowchart for the four groups of defective edge detection programs: (a), (b), and (c) represent the lower-right edge of the bamboo batten detection program, lower-left/upper-right edge of the bamboo batten detection program, and the upper-left edge of bamboo batten detection program, respectively.

\section{System Performance Simulation Test}

To determine the difference between the defective edge detection system and manual detection of defective edges in bamboo battens, a random sample test was carried out. The experiment was conducted from March 5 to March 26, 2017, 
at the Guizu Bamboo Industry Co., Ltd in Guilin, Guangxi Province in Ziyuan County. The defective edge detection experimental device developed at the Guangxi Normal University for automatic sorting of long bamboo batten, as shown in Fig. 9, was used.

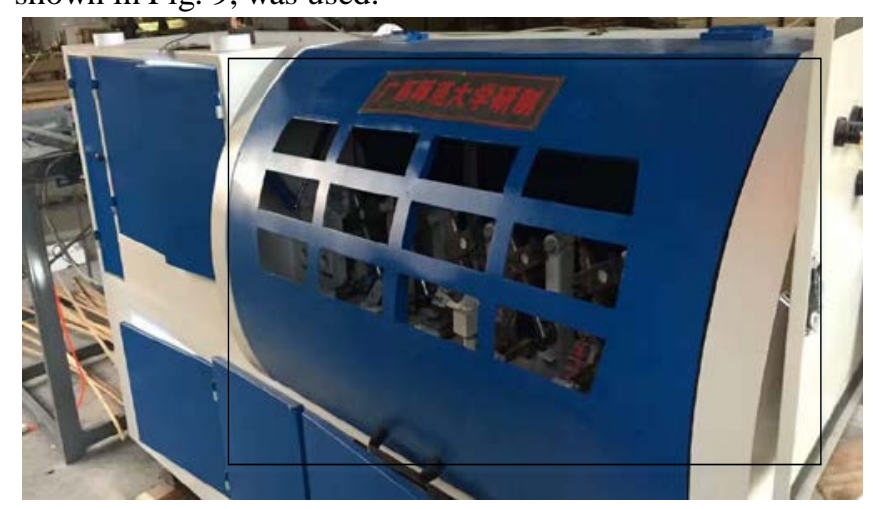

Figure 9. Automatic sorting system

\section{A. Preparation for Testing}

One thousand five hundred randomly screened bamboo battens from the above company were selected and labeled. A set of 300 roots was used each time. First, workers detected the defective edges of the bamboo battens, and then the device detected the defective edges of the bamboo battens. Then, both results were compared. P was used as a production performance indicator, with $\mathrm{P}>95 \%$ indicating the ability to satisfy the production performance requirements. The formula is as follows:

$$
\mathrm{P}=(\mathrm{Z}-|\mathrm{U}-\mathrm{B}|-|\mathrm{U}-\mathrm{A}|) / \mathrm{Z} \times 100 \%
$$

where $\mathrm{Z}, \mathrm{U}, \mathrm{B}$, and $\mathrm{A}$ are the total number of tests, number for which mechanical detection and manual detection showed the bamboo batten to be defective, number detected mechanically as defective, and number detected by workers as defective, respectively.

\section{B. Test Results and Analysis}

The results of the test were analyzed intuitively (Table II), and a success rate line chart for the defective edge detection system was generated (Fig. 10).

TABLE II. RESUlt OF COMPARISON OF MECHANICAL TEST AND WORKERS' TEST

\begin{tabular}{|c|c|c|c|c|c|}
\hline $\mathbf{S} / \mathbf{N}$ & $\mathbf{Z}$ & $\mathbf{A}$ & $\mathbf{B}$ & $\mathbf{U}$ & $\mathbf{P}(\mathbf{\%})$ \\
\hline 1 & 300 & 23 & 27 & 20 & 95.33 \\
\hline 2 & 300 & 46 & 51 & 43 & 96.33 \\
\hline 3 & 300 & 15 & 17 & 9 & 95.33 \\
\hline 4 & 300 & 12 & 12 & 7 & 96.67 \\
\hline 5 & 300 & 21 & 23 & 15 & 95.33 \\
\hline
\end{tabular}

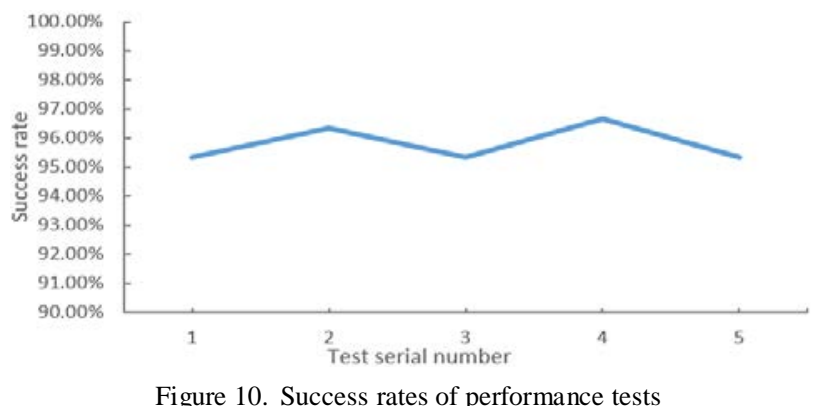

\section{CONCLUSION}

In this paper, a defective edge detection system for bamboo battens was presented. In the device, an optical fiber amplifier was used to detect the intensity of light leakage from the crevices between the contact plate and the edges of bamboo batten. Depending on the intensity, a determination was then made as to whether to send a feedback signal indicating the defective edge of the bamboo by setting the threshold value of the fiber amplifier. The feedback signal of the fiber amplifier is detected by a master controller that then ascertains whether there is a defective edge in the bamboo batten. A method for detecting four edges of two bamboo battens was also presented. Finally, the reliability of the system was verified via a series of experiments.

\section{ACKNOWLEDGMENT}

This research is supported by the "Technological Integration and Demonstration of Full-Procedure BambooBoard-Chopping Mechanized Production Line” Project, sponsored by the Key Project of China Spark Program (Grant No. 2015GA790002).

\section{REFERENCES}

[1] X. Wang, D. Liang, and W. Deng, "Surface grading of bamboo strips using multi-scale color texture features in eigenspace," Computers and Electronics in Agriculture, vol. 73, no. 1, pp. 91-98, 2010.

[2] Q. Xiansheng, S. Xin, L. Qiong, et al., "Online detection and sorting system of bamboo strip based on visual servo," IEEE International Conference on Industrial Technology, 2009, pp. 1-5.

[3] L. P. He, S. X. Song, L. Liu, et al., "Design of bamboo strip detection system based on LabVIEW and MATLAB mixed programming," Applied Mechanics and Materials, vol. 302, pp. 772-775, 2013.

[4] X. Song, X. S. Qin, Q. Liu, et al., "Research and implementation on selection process of bamboo strip based on machine vision," Computer Engineering and Applications, 2006.

[5] Q. Xiansheng, H. Feng, L. Qiong, et al., "Online defect inspection algorithm of bamboo strip based on computer vision," IEEE International Conference on Industrial Technology. IEEE Xplore, 2009, pp. 1-5.

[6] C. H. Zeng, H. Chen, Y. Gao, et al., "Bamboo color grading method based on SVM and color moment," Hubei Agricultural Sciences, vol. 49, no. 2, pp. 455-457, 2010.

[7] L. Qiong, Y. Yanpu, and Z. Yaguang, "A defect detection algorithm for bamboo wood based on wavelet decomposition and clustering analysis," Journal of Computational and Theoretical Nanoscience, 2016.

[8] H. Q. Yang, "The laser rapid detection method and proprietary device for worm-eat defective chopsticks" CN, CN 103293161 A[P]. 2013. 